\title{
Attributes of a Vibration Isolator Design with Stiffness Nonlinearities
}

\author{
Sudhir Kaul \\ School of Engineering and Technology, Western Carolina University, Cullowhee, NC, 28723.
}

(Received 4 August 2017; accepted 26 January 2018)

\begin{abstract}
Inclusion of stiffness nonlinearity in a vibration isolator has been shown to exhibit some advantages such as an increase in the frequency range of isolation. In some engineering applications, it is common to design the vibration isolator such that the stiffness in one direction is significantly different from the stiffness in other directions. Such a design is commonly used for vibration isolators in applications where the packaging and performance requirements along different axes are drastically different. One such example is the vibration isolator for a motorcycle powertrain. This study proposes a design that incorporates stiffness nonlinearities into the vibration isolator along two axes to complement the spring-damper system along the axis of displacement of the single degree-of-freedom system. These stiffness nonlinearities are incorporated into the Maxwell-Voigt (MV) and Maxwell-Maxwell-Voigt (MMV) models for elastomeric isolators. The proposed design is expected to increase the range of vibration isolation and allow some design flexibility in placing the natural frequency of the system while satisfying the specific requirements of a range of products. Results from all the models investigated in this study indicate that adding stiffness nonlinearity, in the form of spring elements along the non-isolating axes, can provide a designer with additional flexibility in placing the natural frequency of the isolation system while enhancing the overall isolation range.
\end{abstract}

\section{NOMENCLATURE}

$\begin{array}{ll}F & \begin{array}{l}\text { Amplitude of external excitation force. } \\ X\end{array} \\ Y, Y_{1}, Y_{2} & \begin{array}{l}\text { Amplitude of base displacement. } \\ \text { Amplitude of displacement of the isolated } \\ \text { mass and the nodes of the Maxwell } \\ \text { elements. }\end{array} \\ \varphi, \varphi_{1}, \varphi_{2} & \begin{array}{l}\text { Phase angles for the displacement of the } \\ \text { mass and the nodes. }\end{array} \\ k_{h}, k_{h x}, k_{h z} & \begin{array}{l}\text { Stiffness of horizontal spring element. } \\ \text { Free length and compressed length of }\end{array} \\ l_{0}, l & \begin{array}{l}\text { spring element. } \\ \text { Base displacement. }\end{array} \\ x(t) & \begin{array}{l}\text { External excitation force. } \\ f_{y}\end{array} \\ y, y_{1}, y_{2} & \begin{array}{l}\text { Displacement of the isolated mass and } \\ \text { the nodes of the Maxwell elements. }\end{array} \\ c_{0}, c_{1}, c_{2} & \text { Damping constants. } \\ k_{0}, k_{1}, k_{2} & \text { Stiffness constants. } \\ m & \text { Mass. }\end{array}$

\section{INTRODUCTION}

The use of passive vibration isolators is widespread in multiple engineering applications. ${ }^{1}$ Designers have been increasingly investigating the use of stiffness and damping nonlinearities to overcome some of the constraints posed by linear vibration isolators. $^{2-4}$ These nonlinearities are specifically incorporated as per design intent; however, there are multiple aspects of a passive elastomeric isolator such as cyclical softening, temperature dependent behavior, etc. that make the behavior inherently nonlinear. ${ }^{1}$ Nonlinearities that have been investigated in the literature include attributes resulting from smart material elements, X-shape structured lever-type design, scissor-like structured platform, etc. ${ }^{1,5,6}$ Passive isolators exhibit a complex behavior that needs to be modeled in order to accurately predict system response before undertaking detailed design of the isolation system. There are multiple models with varying characteristics in the existing literature that have been used to represent specific features of a vibration isolation system, one such example is the use of Maxwell-Voigt and Maxwell Ladder models for multi-degree-of-freedom isolation systems. ${ }^{7}$

Some of the recent literature on nonlinear vibration isolation includes discussion on negative stiffness mechanisms, ${ }^{8,9}$ quasi-zero-stiffness, ${ }^{10,11}$ high-static-low-dynamic stiffness, ${ }^{12}$ among other possible designs that take advantage of nonlinear behavior. The negative frequency mechanism is reported to increase the frequency range of vibration isolation, ${ }^{8}$ and such a mechanism is also found to significantly mitigate the response at resonance. ${ }^{9}$ The quasi-zero-stiffness design is found to be particularly beneficial for low frequency response, ${ }^{10}$ while a multi-direction quasi-zero-stiffness isolator is found to improve the isolation effect in multiple directions simultaneously in addition to providing design flexibility. ${ }^{11}$

Nonlinear designs and nonlinear design attributes have been found to be particularly appealing since they can offer design flexibility in a passive vibration isolator and allow the isolation system to overcome some of the trade-offs associated with the use of a passive isolator. Nonlinearities have been specifically used for designing high-static-low-dynamic stiffness isolators, ${ }^{12}$ for modeling hysteretic behavior, ${ }^{13}$ etc. Many quasi-zero-stiffness isolators have been observed to possess high-static-low-dynamic stiffness characteristics, the existing literature provides examples of isolator designs with both characteristics. ${ }^{12}$ Alternative models have also been proposed in the literature to identify the viscoelastic behavior of an elastomeric isolator by developing a generalized Maxwell model, ${ }^{14}$ or by developing a constitutive model that repre- 


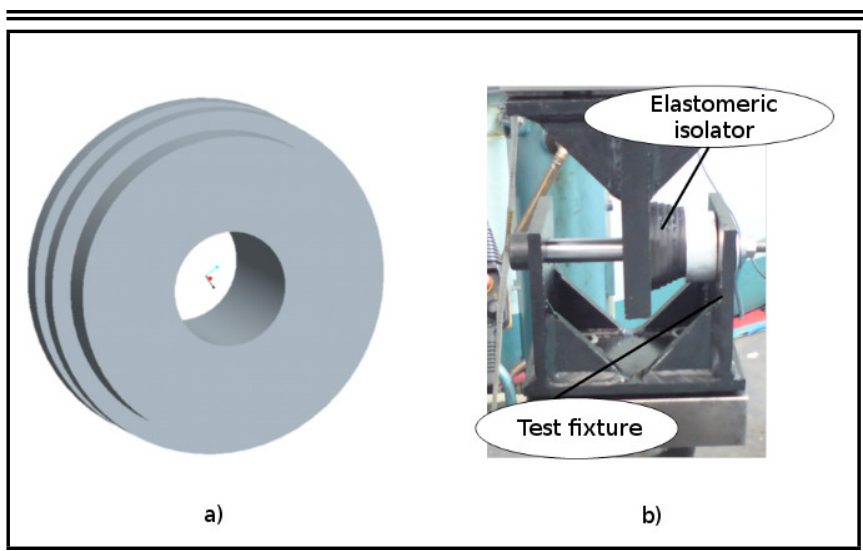

Figure 1. Passive vibration isolators: a) multi-axial vibration isolator, b) multi-axial isolator in shear with test fixture.

sents pre-deformation as well as frequency and amplitude dependence of viscoelastic material. ${ }^{15}$ The constitutive model is shown to be applicable to relatively small as well as large strain amplitudes. ${ }^{15}$ Mechanical designs such as a scissor-like design have also been investigated in the literature for vibration isolation, the scissor-like design is seen to possess inherent nonlinear characteristics and simulations indicate that this design is capable of enhancing some of the isolation characteristics while allowing a designer an ability to adjust stiffness and damping characteristics. ${ }^{6,16}$ Optimization algorithms have often been used to determine adequate parameters of an isolation system to mitigate vibrational response, the use of optimization is useful due to a high number of design variables and constraints associated with the isolation system. ${ }^{17,18}$ Most common design variables that have been used for optimization include stiffness, damping, isolator location, loss factor, elastomer geometry, etc.

In some applications, the vibration isolator is required to exhibit significantly different stiffness and damping properties along different axes in order to meet multiple design criteria. Two such commercially available passive isolators are shown in Fig. 1, the radial stiffness of these isolators is significantly lower than the axial stiffness. The design presented in this study incorporates stiffness nonlinearities into the vibration isolator along two axes to complement the spring-damper system along the third (isolating) axis, which is the axis of displacement of the single degree-of-freedom system.

The design proposed in this paper is expected to augment the tri-axial model commonly used in the existing literature to represent an isolator for three-dimensional models. ${ }^{17}$ This is specifically because the tri-axial model assumes that the stiffness along multiple axes of a passive isolator is independent. Assuming independence of the three axes of stiffness may be accurate only when three separate isolators are being used that are not interconnected in any way. The model presented in this paper, instead, is suitable for any isolator with a threedimensional geometry that is used for vibration isolation in one direction while supporting a six degree-of-freedom system in space. The model developed in this study incorporates spring elements to represent pre-compression as well as relatively higher stiffness along the non-isolating axes. Analysis results from a few different models of the proposed design are presented by using two methods for analysis. The main contribution of this study is the presentation of a model that would be useful for applications in which significantly different stiffness parameters are required in different planes to satisfy performance criteria. The models proposed in this study demonstrate an enhancement of the frequency range of vibration isolation while allowing a designer some more control in placing the natural frequencies of the isolated system and satisfying the specific requirements of a range of products that use passive vibration isolators. The proposed models are presented in Section 2 and simulation results are discussed in Section 3. Overall conclusions are presented in Section 4.

\section{MODEL}

The governing equations of motion (EOM) for the models presented in this section are nonlinear; as a result, two methods have been used for analysis. The first method is the Harmonic Balance Method (HBM), with the underlying assumption that a harmonic input yields a harmonic output that may contain one or more harmonics. ${ }^{19,20} \mathrm{HBM}$ is used as the primary method for analysis in this study. HBM has been used in multiple studies in the literature for the analysis of nonlinear vibration isolation, one such example is the analysis of cubic damping in a vibration isolator. ${ }^{21}$ For all the derivations from the HBM in this section, only the first harmonic is used and the higher order harmonics have been ignored. The system of equations has been derived by substituting for the higher powers of trigonometric ratios and also by making use of the Binomial theorem. The second method used for computing the system response is numerical, using a variable-step, variable-order solver, and has been used to check the validity of the solution. The numerical method has been primarily used for computing the time response.

The first model presented in this section for a single degreeof-freedom (DOF) system incorporates a horizontal spring element into the Maxwell-Voigt (MV) model. A generalized model with multiple Maxwell elements and multiple spring elements is shown in Fig. 2. Similar models such as the Generalized-2 Maxwell model have been used in the literature to represent the viscoelastic behavior of elastomeric isolators. $^{22}$ For the MV model with horizontal spring elements, $k_{2}=c_{2}=0$ (also $y_{2}=0$ ), $k_{h z}=0$, and $k_{h x}=k_{h}$ while using the generalized model in Fig. 2. In Fig. 2, the vertical (y) axis represents the direction of motion and the governing EOM for this model are derived as follows:

$$
\begin{gathered}
m \ddot{y}+k_{0} y+c_{0} \dot{y}+k_{1}\left(y-y_{1}\right)+2 k_{h}\left(1-\frac{l_{0}}{\sqrt{l^{2}+y^{2}}}\right) y=f_{y} \\
k_{1}\left(y-y_{1}\right)=c_{1} \dot{y}_{1}
\end{gathered}
$$

In Eqs. (1) and (2), $k_{0}$ and $c_{0}$ are the spring and damping constants in the vertical $(y)$ direction of motion, $y_{1}$ is the displacement at the node of the Maxwell element. In Eq. (1), $k_{h}$ is the stiffness of the horizontal spring element, $l$ is the compressed length of the spring, and $l_{0}$ is the free-length of the horizontal spring before assembly. Furthermore, $m$ is the mass of the single DOF system and $f_{y}$ is the external excitation force. In Eq. (2), $k_{1}$ and $c_{1}$ are the spring and damping constants of the Maxwell element in the MV model. 


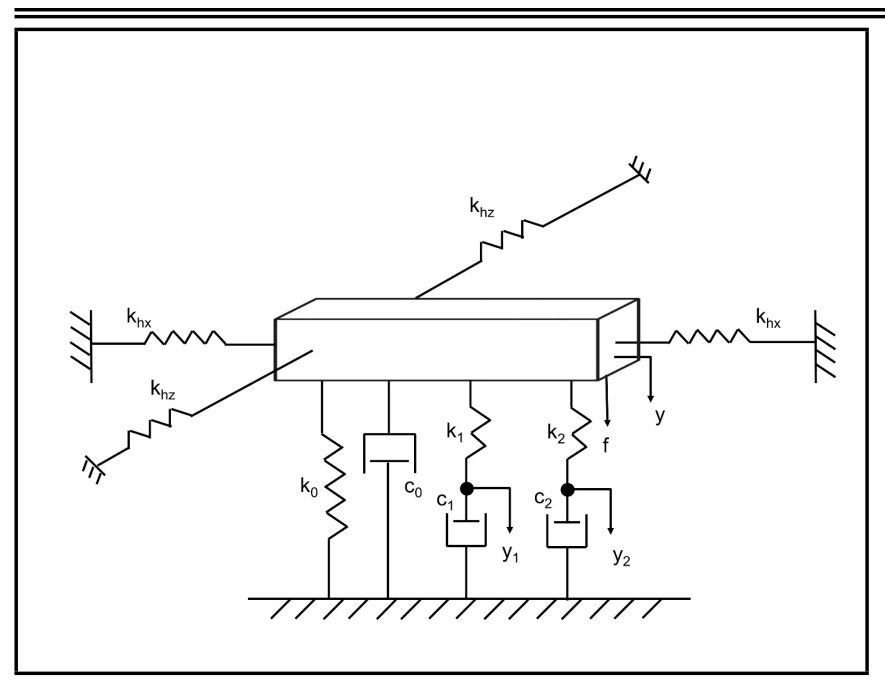

Figure 2. Generalized MMV model with multi-axial spring elements.

Using the Binomial theorem, the first equation-of-motion from Eq. (1) can be expressed as:

$$
\begin{array}{r}
m \ddot{y}+k_{0} y+c_{0} \dot{y}+k_{1}\left(y-y_{1}\right) \\
+2 k_{h}\left[1-\frac{l_{0}}{l}\left(1-\frac{1}{2} \frac{y^{2}}{l^{2}}+\frac{3}{8} \frac{y^{4}}{l^{4}}-\frac{5}{16} \frac{y^{6}}{l^{6}}+\cdots\right)\right] y=f_{y} .
\end{array}
$$

In Eq. (3), higher order terms have been ignored. Furthermore, it may be noted that Eq. (3) holds for $-l^{2}<y^{2}<l^{2}$, this is a reasonable assumption since the response of the system is expected to be small and can be verified from the simulation results in Section 3. Using the HBM, for a sinusoidal input of $f_{y}=F \sin (\omega t-\varphi)$ with an amplitude $F$ and phase $\varphi$, the output displacements are $y=Y \sin \omega t$ with an amplitude $Y$ and $y_{1}=Y_{1} \sin \left(\omega t-\varphi_{1}\right)$ with an amplitude $Y_{1}$ and a phase of $\varphi_{1}$. Substituting these expressions in Eq. (3) and equating the sine and cosine coefficients between the two sides from Eq. (2) and Eq. (3) yields the following:

$$
\begin{array}{r}
-m \omega^{2} Y+k_{0} Y+k_{1} Y-k_{1} Y_{1} \cos \varphi_{1} \\
+2 k_{h}\left[1-\frac{l_{0}}{l}\left(1-\frac{1}{2} \frac{Y^{2}}{l^{2}} \frac{1}{2}+\frac{3}{8} \frac{Y^{4}}{l^{4}} \frac{3}{8}-\frac{5}{16} \frac{Y^{6}}{l^{6}} \frac{5}{16}\right)\right] Y \\
=F \cos \varphi
\end{array}
$$

$$
\begin{gathered}
c_{0} \omega Y+k_{1} Y_{1} \sin \varphi_{1}=-F \sin \varphi ; \\
c_{1} \omega Y_{1} \sin \varphi_{1}=k_{1} Y-k_{1} Y_{1} \cos \varphi_{1} ; \\
c_{1} \omega Y_{1} \cos \varphi_{1}=k_{1} Y_{1} \sin \varphi_{1} .
\end{gathered}
$$

The system of equations in Eq. (4) results from the comparison of first harmonic coefficients from Eq. (2) and Eq. (3). It may be noted that all higher order harmonics have been ignored.

For the Maxwell-Maxwell-Voigt (MMV) model in conjunction with a horizontal spring element, Fig. 2 can be used for reference with $k_{h z}=0$ and $k_{h x}=k_{h}$. It may be noted that the use of two Maxwell elements has been found to enhance the capability of the model by allowing the model to predict dynamic stiffness and loss angle through the entire frequency range. ${ }^{22}$ The governing EOM for this model are as follows:

$$
\begin{gathered}
m \ddot{y}+k_{0} y+c_{0} \dot{y}+k_{1}\left(y-y_{1}\right)+k_{2}\left(y-y_{2}\right) \\
+2 k_{h}\left(1-\frac{l_{0}}{\sqrt{t^{2}+y^{2}}}\right) y=f_{y} \\
k_{1}\left(y-y_{1}\right)=c_{1} \dot{y}_{1} ; \\
k_{2}\left(y-y_{2}\right)=c_{2} \dot{y}_{2} ;
\end{gathered}
$$

In Eq. (5), $y_{1}$ and $y_{2}$ are the displacements at the two nodes of the Maxwell elements shown in Fig. 2. Using similar steps to the ones used for the MV model along with the use of the Binomial theorem and the HBM, the following system of equations can be derived for the MMV model with horizontal spring elements:

$$
\begin{gathered}
-m \omega^{2} Y+k_{0} Y+k_{1} Y-k_{1} Y_{1} \cos \varphi_{1}+k_{2} Y-k_{2} Y_{2} \cos \varphi_{2} \\
+2 k_{h}\left[1-\frac{l_{0}}{l}\left(1-\frac{1}{2} \frac{Y^{2}}{l^{2}} \frac{1}{2}+\frac{3}{8} \frac{Y^{4}}{l^{4}} \frac{3}{8}-\frac{5}{16} \frac{Y^{6}}{l^{6}} \frac{5}{16}\right)\right] Y \\
=F \cos \varphi ; \\
c_{0} \omega Y+k_{1} Y_{1} \sin \varphi_{1}+k_{2} Y_{2} \sin \varphi_{2}=-F \sin \varphi ; \\
c_{1} \omega Y_{1} \sin \varphi_{1}=k_{1} Y-k_{1} Y_{1} \cos \varphi_{1} ; \\
c_{1} \omega Y_{1} \cos \varphi_{1}=k_{1} Y_{1} \sin \varphi_{1} ; \\
c_{2} \omega Y_{2} \sin \varphi_{2}=k_{2} Y-k_{2} Y_{2} \cos \varphi_{2} \\
c_{2} \omega Y_{2} \cos \varphi_{2}=k_{2} Y_{2} \sin \varphi_{2} .
\end{gathered}
$$

It may be noted that the following responses have been assumed for the rigid body and the two nodes to derive the system in Eq. (8): $y=Y \sin \omega t, y_{1}=Y_{1} \sin \left(\omega t-\varphi_{1}\right)$, $y_{2}=Y_{2} \sin \left(\omega t-\varphi_{2}\right)$, for an input of $f_{y}=F \sin (\omega t-\varphi)$. In Eq. (8), $Y_{1}$ and $Y_{2}$ are the displacement amplitudes of the two nodes respectively, and $\varphi_{1}$ and $\varphi_{2}$ are the phase angles associated with the motion of the two nodes respectively.

The MV model with two horizontal stiffness elements along $x$ and $z$ axes is specifically used to incorporate the need for different stiffness properties along the non-isolating axes. Figure 2 can be used for reference with $k_{2}=c_{2}=0$ (also $y_{2}=0$ ). The governing EOM for this model are derived to be as follows:

$$
\begin{gathered}
m \ddot{y}+k_{0} y+c_{0} \dot{y}+k_{1}\left(y-y_{1}\right) \\
+2 k_{h x}\left(1-\frac{l_{0 x}}{\sqrt{l_{x}^{2}+y^{2}}}\right) y+2 k_{h z}\left(1-\frac{l_{0 z}}{\sqrt{l_{z}^{2}+y^{2}}}\right) y \\
=f_{y} \\
k_{1}\left(y-y_{1}\right)=c_{1} \dot{y}_{1} .
\end{gathered}
$$

In Eq. (9), $k_{h x}$ and $k_{h z}$ represent the stiffness elements along $x$ and $z$ axes, respectively. The free-length of the two springs is $l_{0 x}$ and $l_{0 z}$, and $l_{x}$ and $l_{z}$ are the compressed lengths of the two springs respectively at assembly. The rest of the variables in Eq. (9) and Eq. (10) are identical to the other MV model in Fig. 2. 
The following system of equations is derived for the MV model with two horizontal stiffness elements by using the HBM:

$$
\begin{array}{r}
-m \omega^{2} Y+k_{0} Y+k_{1} Y-k_{1} Y_{1} \cos \varphi_{1} \\
+2 k_{h x}\left[1-\frac{l_{0 x}}{l_{x}}\left(1-\frac{1}{2} \frac{Y^{2}}{l_{x}^{2}} \frac{1}{2}+\frac{3}{8} \frac{Y^{4}}{l_{x}^{4}} \frac{3}{8}-\frac{5}{16} \frac{Y^{6}}{l_{x}^{6}} \frac{5}{16}\right)\right] Y \\
+2 k_{h z}\left[1-\frac{l_{0 z}}{l_{z}}\left(1-\frac{1}{2} \frac{Y^{2}}{l_{z}^{2}} \frac{1}{2}+\frac{3}{8} \frac{Y^{4}}{l_{z}^{4}} \frac{3}{8}-\frac{5}{16} \frac{Y^{6}}{l_{z}^{6}} \frac{5}{16}\right)\right] Y \\
=F \cos \varphi
\end{array}
$$

$$
\begin{gathered}
c_{0} \omega Y+k_{1} Y_{1} \sin \varphi_{1}=-F \sin \varphi \\
c_{1} \omega Y_{1} \sin \varphi_{1}=k_{1} Y-k_{1} Y_{1} \cos \varphi_{1} \\
c_{1} \omega Y_{1} \cos \varphi_{1}=k_{1} Y_{1} \sin \varphi_{1} .
\end{gathered}
$$

The last model investigated in this study is represented by the complete model shown in Fig. 2, this model consists of two horizontal stiffness elements along the $x$ and $z$ directions incorporated into the MMV model. The derived EOM for this model are as follows:

$$
\begin{gathered}
m \ddot{y}+k_{0} y+c_{0} \dot{y}+k_{1}\left(y-y_{1}\right)+k_{2}\left(y-y_{2}\right) \\
+2 k_{h x}\left(1-\frac{l_{0 x}}{\sqrt{l_{x}^{2}+y^{2}}}\right) y+2 k_{h z}\left(1-\frac{l_{0 z}}{\sqrt{l_{z}^{2}+y^{2}}}\right) y \\
=f_{y} \\
k_{1}\left(y-y_{1}\right)=c_{1} \dot{y}_{1} \\
k_{2}\left(y-y_{2}\right)=c_{2} \dot{y}_{2} .
\end{gathered}
$$

For this model, using the HBM for the EOM in Eqs. (12), (13) and (14) yields the following system of equations:

$$
\begin{array}{r}
-m \omega^{2} Y+k_{0} Y+k_{1} Y-k_{1} Y_{1} \cos \varphi_{1}+k_{2} Y-k_{2} Y_{2} \cos \varphi_{2} \\
+2 k_{h x}\left[1-\frac{l_{0 x}}{l_{x}}\left(1-\frac{1}{2} \frac{Y^{2}}{l_{x}^{2}} \frac{1}{2}+\frac{3}{8} \frac{Y^{4}}{l_{x}^{4}} \frac{3}{8}-\frac{5}{16} \frac{Y^{6}}{l_{x}^{6}} \frac{5}{16}\right)\right] Y \\
+2 k_{h z}\left[1-\frac{l_{0 z}}{l_{z}}\left(1-\frac{1}{2} \frac{Y^{2}}{l_{z}^{2}} \frac{1}{2}+\frac{3}{8} \frac{Y^{4}}{l_{z}^{4}} \frac{3}{8}-\frac{5}{16} \frac{Y^{6}}{l_{z}^{6}} \frac{5}{16}\right)\right] Y \\
=F \cos \varphi
\end{array}
$$

$$
\begin{gathered}
c_{0} \omega Y+k_{1} Y_{1} \sin \varphi_{1}+k_{2} Y_{2} \sin \varphi_{2}=-F \sin \varphi \\
c_{1} \omega Y_{1} \sin \varphi_{1}=k_{1} Y-k_{1} Y_{1} \cos \varphi_{1} ; \\
c_{1} \omega Y_{1} \cos \varphi_{1}=k_{1} Y_{1} \sin \varphi_{1} ; \\
c_{2} \omega Y_{2} \sin \varphi_{2}=k_{2} Y-k_{2} Y_{2} \cos \varphi_{2} ; \\
c_{2} \omega Y_{2} \cos \varphi_{2}=k_{2} Y_{2} \sin \varphi_{2} .
\end{gathered}
$$

The system of equations in Eq. (15) needs to be solved for the displacement amplitude of the rigid body, $Y$, and the displacement amplitudes of the nodes, $Y_{1}$ and $Y_{2}$. The corresponding phase angles, $\varphi, \varphi_{1}$, and $\varphi_{2}$, associated with the input force and the displacement of the two nodes are also calculated from the system of equations in Eq. (15). A nonlinear least-squares based method is used to solve the system of equations derived in this section for all the models. This method is primarily based on Newton's method in conjunction with the Powell Dogleg procedure. ${ }^{23}$ This method is reported to be robust and capable of overcoming problems related to singularities and convergence. ${ }^{23}$

For the MV model with horizontal spring elements along $x$ and $z$ axes, a base excitation of $x=X \sin (\omega t-\varphi)$ is used to compute the displacement transmissibility and the effect of the parameters associated with the horizontal stiffness elements. Substitution of base excitation and the use of the HBM results in the following system of equations for base excitation for this model:

$$
\begin{array}{r}
-m \omega^{2} Y+k_{0} Y+k_{1} Y-k_{1} Y_{1} \cos \varphi_{1} \\
+2 k_{h x}\left[1-\frac{l_{0 x}}{l_{x}}\left(1-\frac{1}{2} \frac{Y^{2}}{l_{x}^{2}} \frac{1}{2}+\frac{3}{8} \frac{Y^{4}}{l_{x}^{4}} \frac{3}{8}-\frac{5}{16} \frac{Y^{6}}{l_{x}^{6}} \frac{5}{16}\right)\right] Y \\
+2 k_{h z}\left[1-\frac{l_{0 z}}{l_{z}}\left(1-\frac{1}{2} \frac{Y^{2}}{l_{z}^{2}} \frac{1}{2}+\frac{3}{8} \frac{Y^{4}}{l_{z}^{4}} \frac{3}{8}-\frac{5}{16} \frac{Y^{6}}{l_{z}^{6}} \frac{5}{16}\right)\right] Y \\
=k_{0} X \cos \varphi+c_{0} \omega X \sin \varphi
\end{array}
$$

$$
\begin{gathered}
c_{0} \omega Y+k_{1} Y_{1} \sin \varphi_{1}=-k_{0} X \sin \varphi+c_{0} \omega X \cos \varphi \\
c_{1} \omega Y_{1} \sin \varphi_{1}-c_{1} \omega X \sin \varphi=k_{1} Y-k_{1} Y_{1} \cos \varphi_{1} \\
c_{1} \omega Y_{1} \cos \varphi_{1}-c_{1} \omega X \cos \varphi=k_{1} Y_{1} \sin \varphi_{1} .
\end{gathered}
$$

All the variables in Eq. (16) are shown in the model in Fig. 1. The displacement transmissibility for this model is the ratio $Y / X$ for a known amplitude, $X$, of the base excitation with the corresponding phase angle being $\varphi$. The system of equations in Eq. (16) is solved for four unknowns $-Y, Y_{1}, \varphi$, and $\varphi_{1}$ for a unit displacement amplitude of base excitation.

For computing the displacement transmissibility for the MMV model with horizontal spring elements along $x$ and $z$ axes, a base excitation of $x=X \sin (\omega t-\varphi)$ yields the following system of equations:

$$
\begin{array}{r}
-m \omega^{2} Y+k_{0} Y+k_{1} Y-k_{1} Y_{1} \cos \varphi_{1}+k_{2} Y-k_{2} Y_{2} \cos \varphi_{2} \\
+2 k_{h x}\left[1-\frac{l_{0 x}}{l_{x}}\left(1-\frac{1}{2} \frac{Y^{2}}{l_{x}^{2}} \frac{1}{2}+\frac{3}{8} \frac{Y^{4}}{l_{x}^{4}} \frac{3}{8}-\frac{5}{16} \frac{Y^{6}}{l_{x}^{6}} \frac{5}{16}\right)\right] Y \\
+2 k_{h z}\left[1-\frac{l_{0 z}}{l_{z}}\left(1-\frac{1}{2} \frac{Y^{2}}{l_{z}^{2}} \frac{1}{2}+\frac{3}{8} \frac{Y^{4}}{l_{z}^{4}} \frac{3}{8}-\frac{5}{16} \frac{Y^{6}}{l_{z}^{6}} \frac{5}{16}\right)\right] Y \\
=k_{0} X \cos \varphi+c_{0} \omega X \sin \varphi ;
\end{array}
$$

$$
\begin{gathered}
c_{0} \omega Y+k_{1} Y_{1} \sin \varphi_{1}+k_{2} Y_{2} \sin \varphi_{2} \\
=-k_{0} X \sin \varphi+c_{0} \omega X \cos \varphi \\
c_{1} \omega Y_{1} \sin \varphi_{1}-c_{1} \omega X \sin \varphi=k_{1} Y-k_{1} Y_{1} \cos \varphi_{1} \\
c_{1} \omega Y_{1} \cos \varphi_{1}-c_{1} \omega X \cos \varphi=k_{1} Y_{1} \sin \varphi_{1} ; \\
c_{2} \omega Y_{2} \sin \varphi_{2}-c_{2} \omega X \sin \varphi=k_{2} Y-k_{2} Y_{2} \cos \varphi_{2} \\
c_{2} \omega Y_{2} \cos \varphi_{2}-c_{2} \omega X \cos \varphi=k_{2} Y_{2} \sin \varphi_{2} .
\end{gathered}
$$

The variables in Eq. (17) correspond to the model in Fig. 1. For this model, displacement transmissibility is calculated by computing $Y / X$ for a unit amplitude of base excitation, $X$, with a displacement of $Y_{1} \sin \left(\omega t-\varphi_{1}\right)$ and $Y_{2} \sin \left(\omega t-\varphi_{2}\right)$ at the two nodes of the MMV model. The system of equations in 


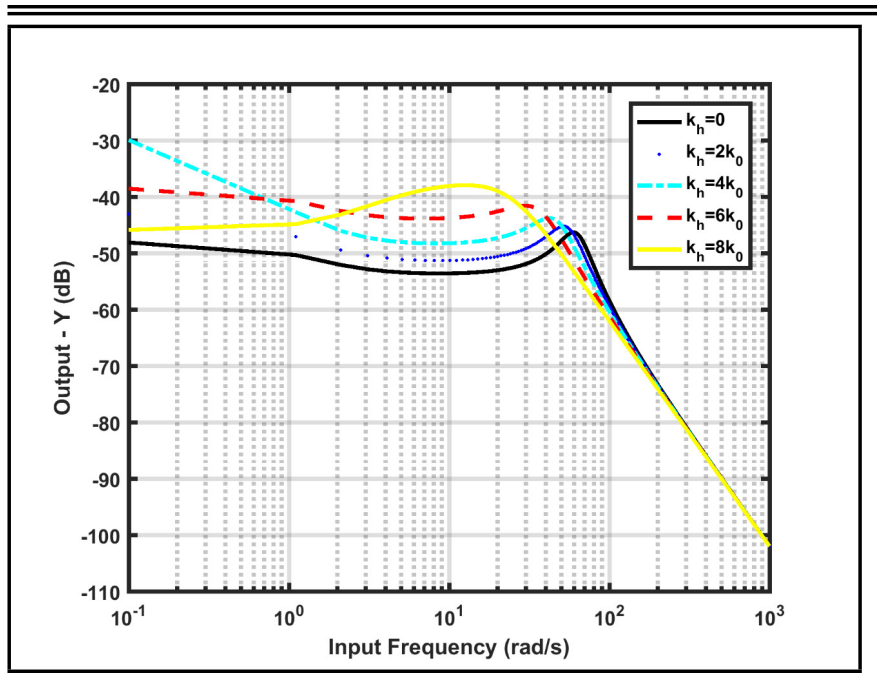

Figure 3. Frequency response - MV Model with one horizontal spring element at $10 \%$ pre-compression.

Eq. (17) is solved for six unknowns $-Y, Y_{1}, Y_{2}, \varphi, \varphi_{1}$, and $\varphi_{2}$ for a unit displacement amplitude of base excitation.

The models presented in this section have been used for multiple simulations in Section 3. All the variables associated with the model are identified by using load-deflection characteristics from a commercially available vibration isolator, shown in Figure 1a, that has been tested for this study.

\section{RESULTS}

The simulation results for the models of the proposed design are presented in this section. Test results from an elastomeric isolator are used to characterize the MV and MMV models along the isolating axis. The vibration isolator shown in Fig. 1a has been used for characterization. The loaddeflection data is collected from a single-axis test for the elastomeric isolator at multiple frequencies, and an optimization program is used to identify the variables associated with each model. It may be noted that the variables associated with the horizontal spring elements have not been determined from model characterization. The variables for the MV model are as follows: $k_{0}=251.26 \mathrm{~N} / \mathrm{mm}, c_{0}=3.23 \mathrm{~N}$ $\mathrm{s} / \mathrm{mm}, k_{1}=237.38 \mathrm{~N} / \mathrm{mm}, c_{1}=121.92 \mathrm{~N}-\mathrm{s} / \mathrm{mm}$. The variables for the MMV model are found to be as follows: $k_{0}=251.26 \mathrm{~N} / \mathrm{mm}, c_{0}=3.23 \mathrm{~N}-\mathrm{s} / \mathrm{mm}, k_{1}=237.38 \mathrm{~N} / \mathrm{mm}$, $c_{1}=121.92 \mathrm{~N}-\mathrm{s} / \mathrm{mm}, k_{2}=180.59 \mathrm{~N} / \mathrm{mm}, c_{2}=1.89 \mathrm{~N}-\mathrm{s} / \mathrm{mm}$. A mass of $125 \mathrm{~kg}$ is used for the single DOF system and a free length of $50 \mathrm{~mm}$ is used for the horizontal spring elements in all the simulations in this section. The three variables associated with the horizontal spring element - stiffness, free length and pre-compression - have been varied in order to understand the influence of these variables on the frequency response as well as the time response.

Figure 3 shows the frequency response of the MV model with one horizontal spring element at varying levels of horizontal stiffness $\left(k_{h}\right)$ for $10 \%$ pre-compression. It may be noted that the output is derived for a sinusoidal input with unit amplitude. Also, $k_{h}=0$ corresponds to a conventional MaxwellVoigt model without any horizontal spring elements.

Increasing horizontal stiffness is seen to result in a slight reduction in the natural frequency, but the response is seen to

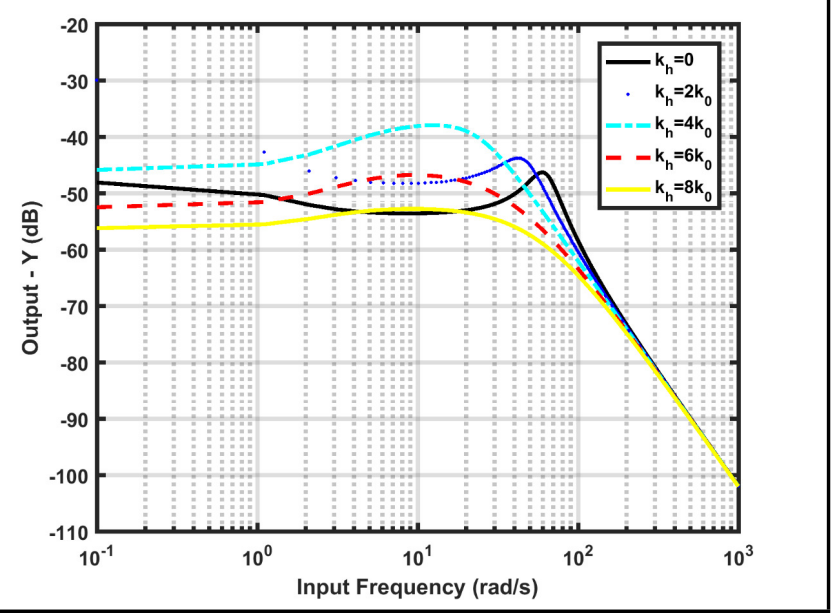

Figure 4. Frequency response - MV Model with two horizontal spring elements at $10 \%$ pre-compression.

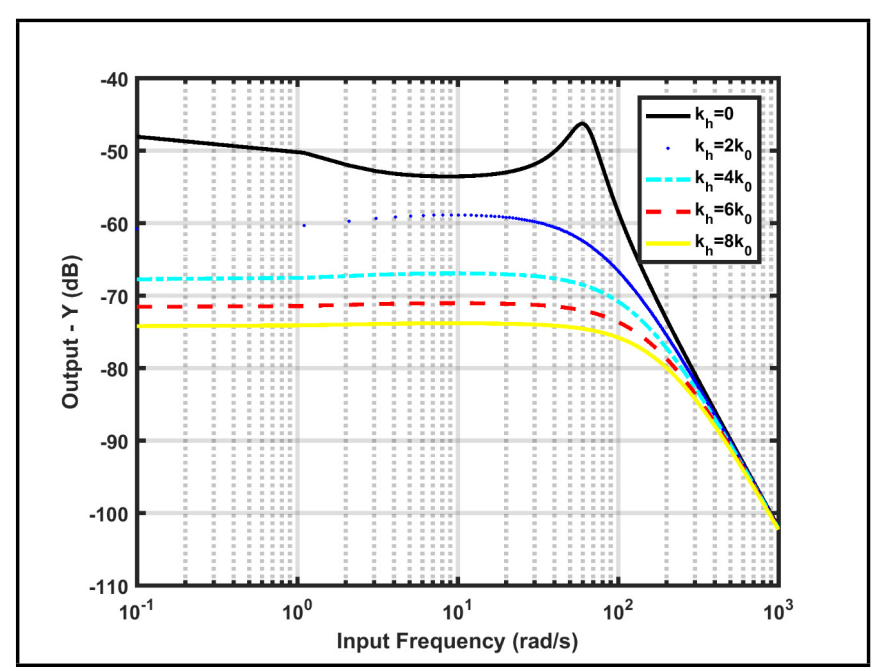

Figure 5. Frequency response - MV Model with two horizontal spring elements at $40 \%$ pre-compression.

increase at lower frequencies without significantly influencing the response at higher frequencies. Also, the peak response shows an increasing trend with an increase in stiffness. However, the response at lower frequencies does not show a trend. Adding a horizontal spring element along another axis to the MV model exhibits similar results for $10 \%$ pre-compression, leading to a reduction in the natural frequency. However, the shift in the natural frequency is seen to be accentuated and the frequency response is seen to significantly reduce at lower frequencies for a substantial increase in stiffness. This can be seen from Fig. 4 for the response of the MV model with two horizontal spring elements along the two non-isolating axes, it can be seen that the response is reduced through the entire frequency range for $k_{h}=8 k_{0}$. It may be noted that $k_{h}$ indicates the stiffness of the springs in both directions ( $x$ and $z$ ), even though the model is capable of accommodating different stiffness along the two non-isolating axes.

Pre-compression of the horizontal spring elements is seen to significantly influence the frequency response, this can be seen from the response in Fig. 5 and Fig. 6. In Fig. 5, increase in stiffness is seen to significantly reduce the response through the entire frequency range at $40 \%$ pre-compression of the two 


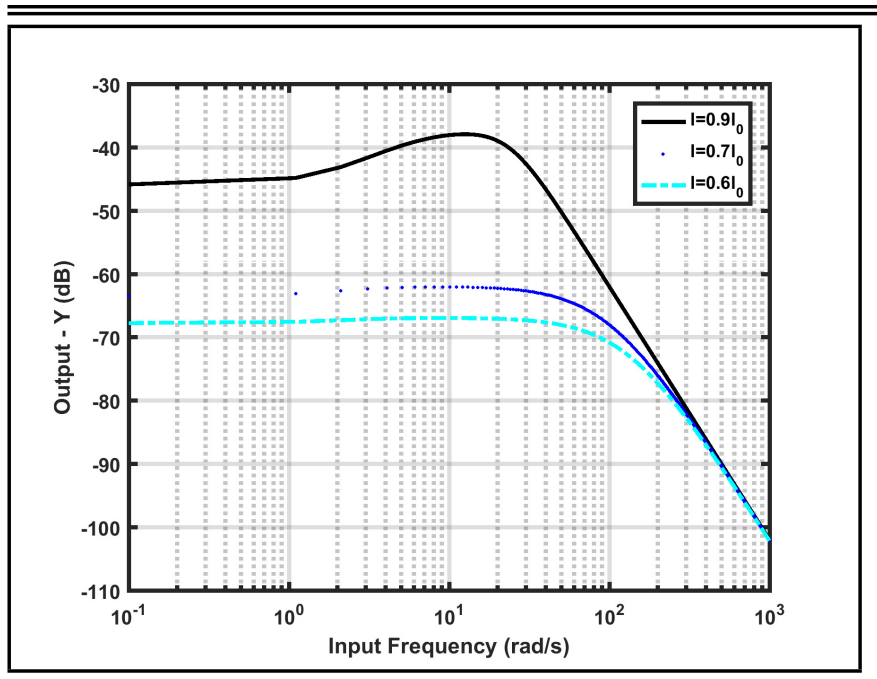

Figure 6. Frequency response at $k_{h}=8 k_{0}-\mathrm{MV}$ Model with one horizontal spring element.

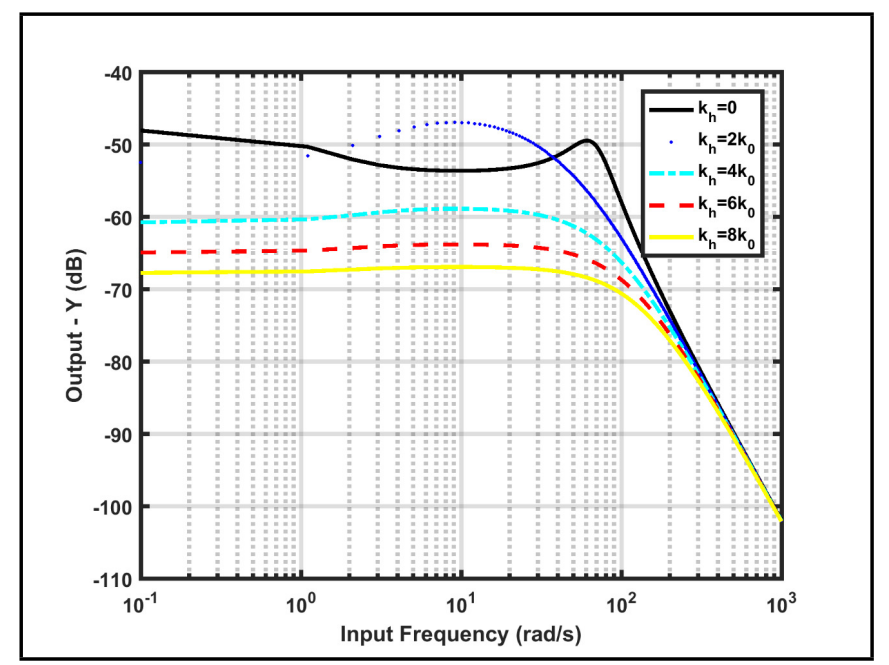

Figure 7. Frequency response - MMV Model with one horizontal spring element at $40 \%$ pre-compression.

horizontal spring elements, this can be directly compared to the results in Fig. 4. This phenomenon can be further observed in Fig. 6 as the pre-compression is changed from $10 \%$ to $40 \%$ at a constant level of horizontal stiffness for the MV model. Overall, the results for the MV model indicate that an incorporation of horizontal stiffness and pre-compression of the horizontal spring elements can be successfully used to adjust the natural frequency of the system and control the response amplitude with limited trade-offs. Particularly, significant benefits are observed at high levels of stiffness in conjunction with high levels of pre-compression.

The MMV model exhibits characteristics that are similar to the observations from the response of the MV model. The MMV model exhibits a significant reduction in response through the entire frequency range with the increasing stiffness of the horizontal elements in conjunction with a relatively higher pre-compression. One such result for the MMV model can be seen in Fig. 7 and Fig. 8 with the amplitude and phase response at $40 \%$ pre- compression. The amplitude response is seen to reduce through the entire frequency range for $k_{h}>2 k_{0}$, as seen in Fig. 7 .

Another result for the MMV model can be seen in Fig. 9

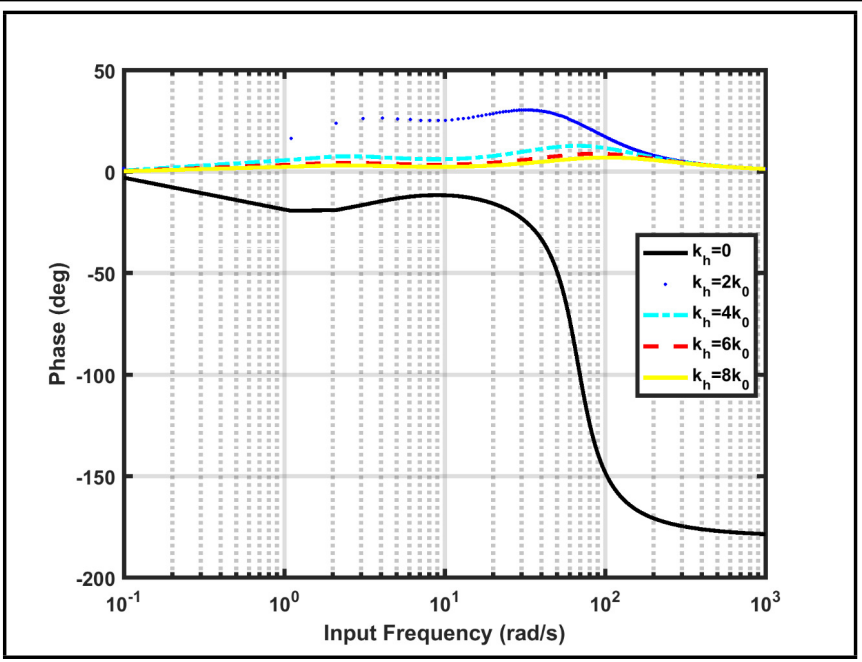

Figure 8. Phase angle - MMV Model with one horizontal spring element at $40 \%$ pre-compression.

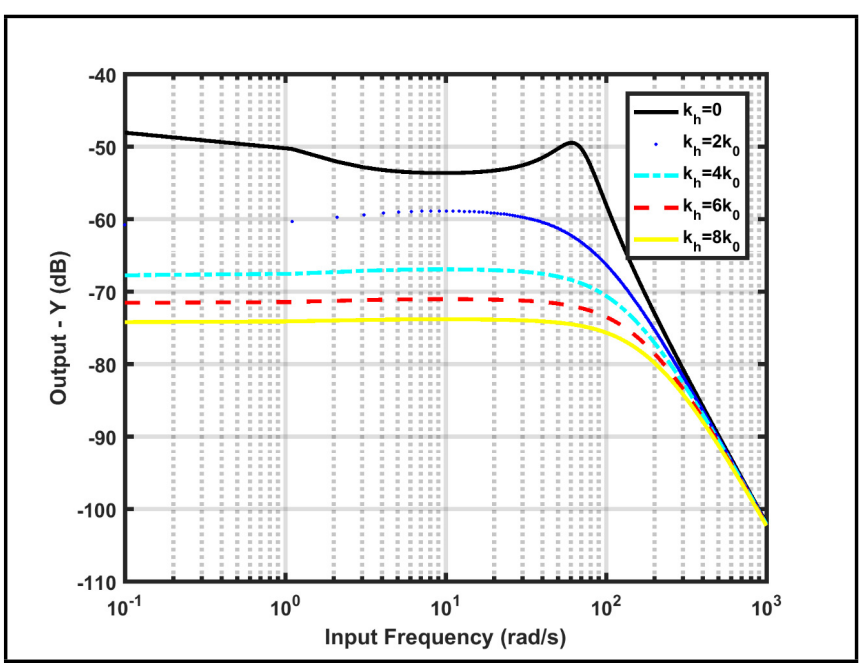

Figure 9. Frequency response - MMV Model with two horizontal spring elements at $40 \%$ pre-compression.

and Fig. 10 for a pre-compression of $40 \%$ with two horizontal spring elements, the response is seen to decrease with increasing stiffness for all levels of horizontal stiffness with limited trade-offs at lower frequencies. The results for the MV and MMV models are similar to the results reported for the Voigt model in the existing literature. ${ }^{2}$ Figure 10 shows the phase angle associated with the response shown in Fig. 9. The phase angle for $k_{h}=0$ is similar to a damped system with a very low damping ratio, but the phase angle remains lower than $20 \mathrm{deg}$. through the entire frequency range for all non-zero values of horizontal stiffness. This indicates that the change in the frequency response resulting from increasing horizontal stiffness or pre-compression is not analogous to an increase in the damping ratio. Instead, the parameters of the horizontal spring elements significantly reduce the phase lag between excitation input and the system response, as seen in Fig. 10.

Time response of the models has been investigated by using a quasi-constant step implementation of the backward difference method. ${ }^{23}$ In the algorithm, the time step is reduced only if convergence is not achieved initially and the Jacobian is updated if the problem is found to be significantly stiff. ${ }^{23}$ The time response has been used to compare the results of the 


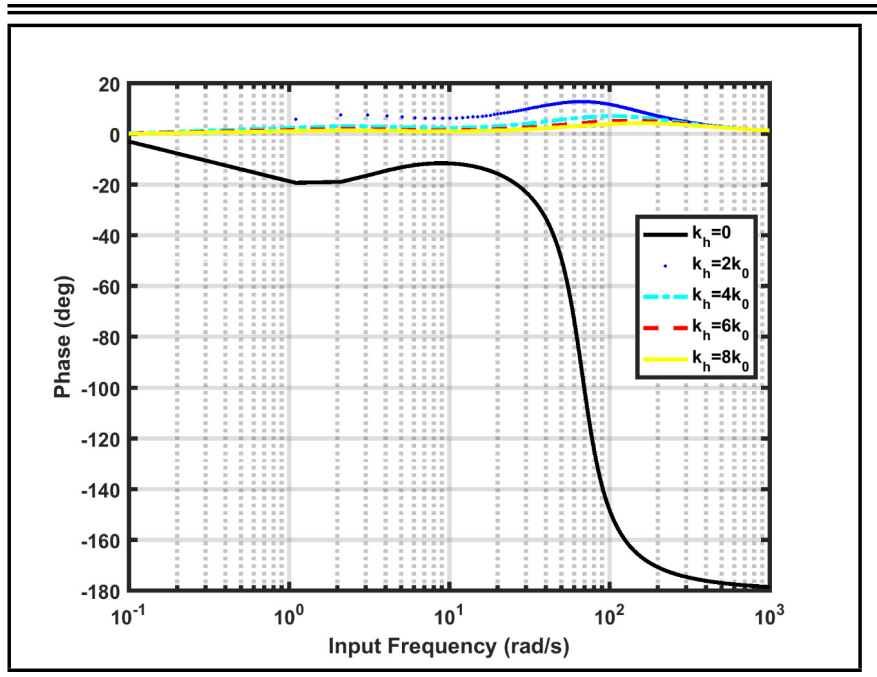

Figure 10. Phase angle - MMV Model with two horizontal spring elements at $40 \%$ pre-compression.

HBM with numerical integration and to determine the step response for the models discussed in this paper. The step response of the MV model with one horizontal spring element at $10 \%$ pre-compression is shown in Fig. 11. An increase in horizontal stiffness is seen to result in an increase in the amplitude of the step response. A similar step response is exhibited by the MMV model with one or two horizontal spring elements. Figure 12 shows the transient force transmitted by the isolator due to a step input acting on the rigid body supported by the isolator. The transmitted force in the transient response is seen to reduce with an increase in horizontal stiffness. All MV and MMV models investigated in this study are seen to exhibit similar trends for transmitted force due to a step input. The trends of the results from the time response correspond to the results seen from the frequency response. This can be observed by comparing the results from Fig. 3 and Fig. 11. For instance, the increase in rise time, as defined by the time taken for the response to reach $90 \%$ of the steady state value, corresponds to a decrease in the natural frequency, as seen from Fig. 3 for increasing horizontal stiffness at the same level of pre-compression. The numerical solution was not able to converge to a solution for higher levels of stiffness, this needs to be investigated further.

The models proposed in this study have also been investigated for base excitation. It can be seen from the results in Fig. 13 and Fig. 14 that an increasing stiffness of the horizontal spring elements results in shifting the peak transmissibility to a lower frequency, and this shift is accompanied by an increase in displacement transmissibility at lower frequencies. However, a substantial increase in stiffness $\left(k_{h}=8 k_{0}\right)$ results in a reduction in displacement transmissibility through the entire frequency range. This is consistent with the results derived from the frequency response.

In order to compare the capability of the models discussed in this study with a commonly used model, the displacement transmissibility of the MV model with two horizontal spring elements at $10 \%$ pre-compression is compared with the results from a Voigt model (one spring and one damper element in parallel along the isolating axis). The results from this comparison are shown in Fig. 15. The advantages of the model proposed in this study are expected to be similar to the quasi-zero-stiffness

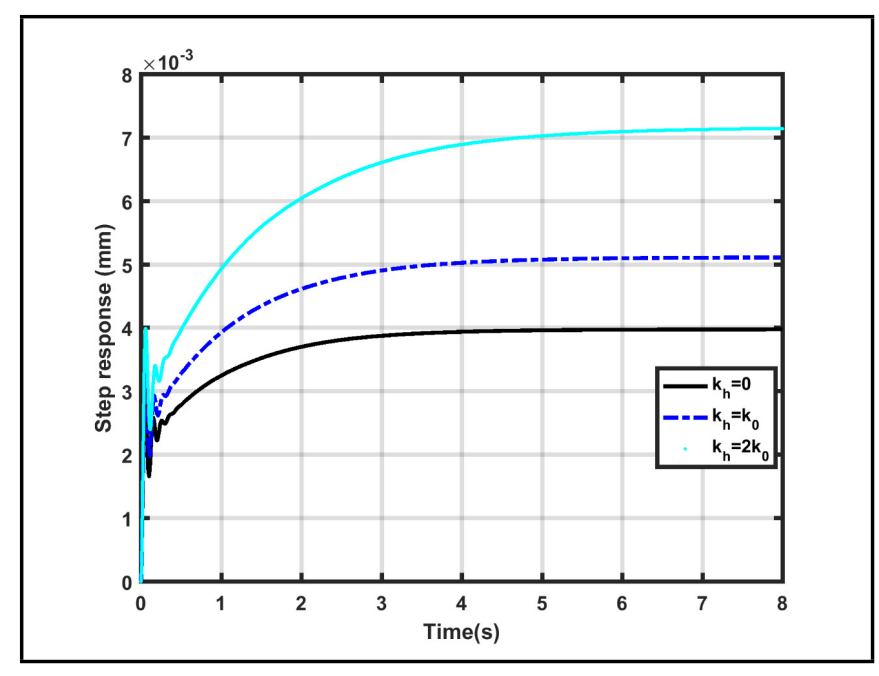

Figure 11. Step response - MV Model with one horizontal spring element at $10 \%$ pre-compression.

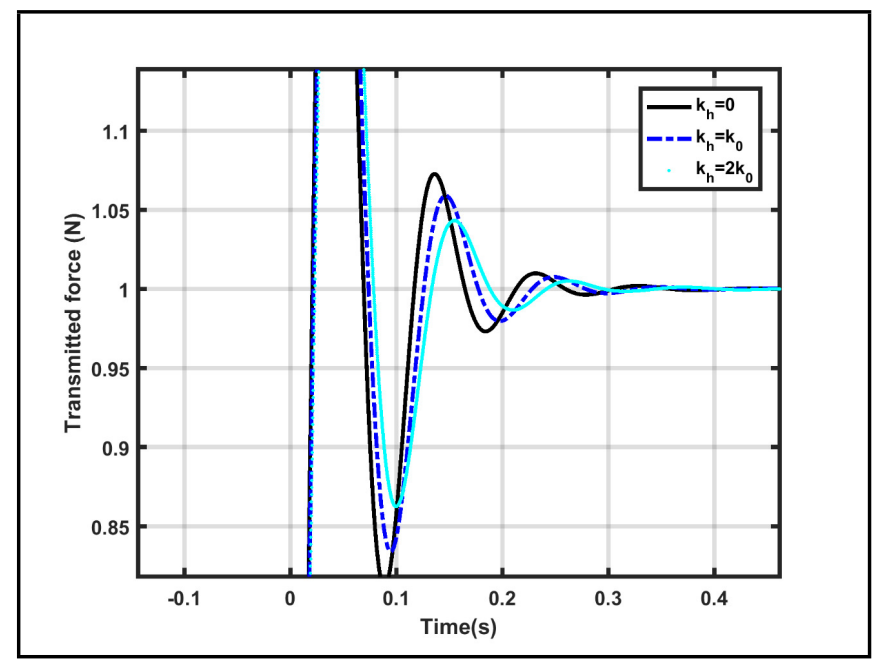

Figure 12. Transmitted force - Step input - MMV Model with one horizontal spring element at $10 \%$ pre-compression.

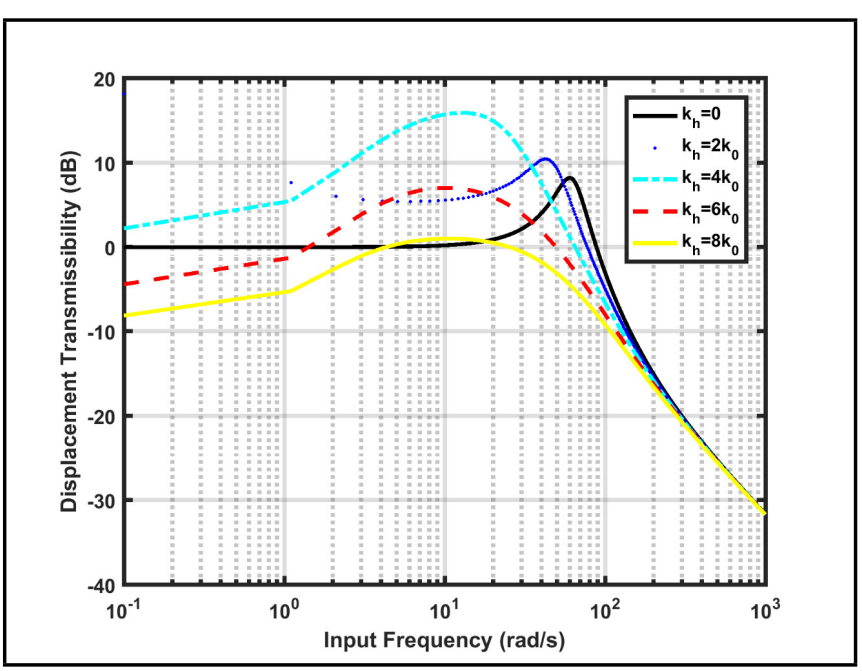

Figure 13. Displacement Transmissibility — MV Model with two horizontal spring elements at $10 \%$ pre-compression. 


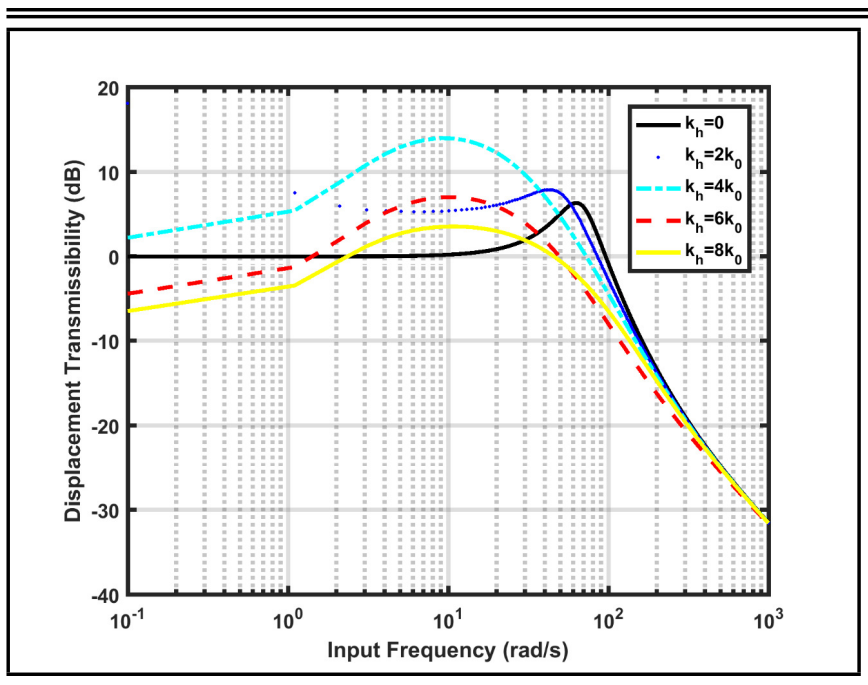

Figure 14. Displacement Transmissibility - MMV Model with two horizontal spring elements at $10 \%$ pre-compression.

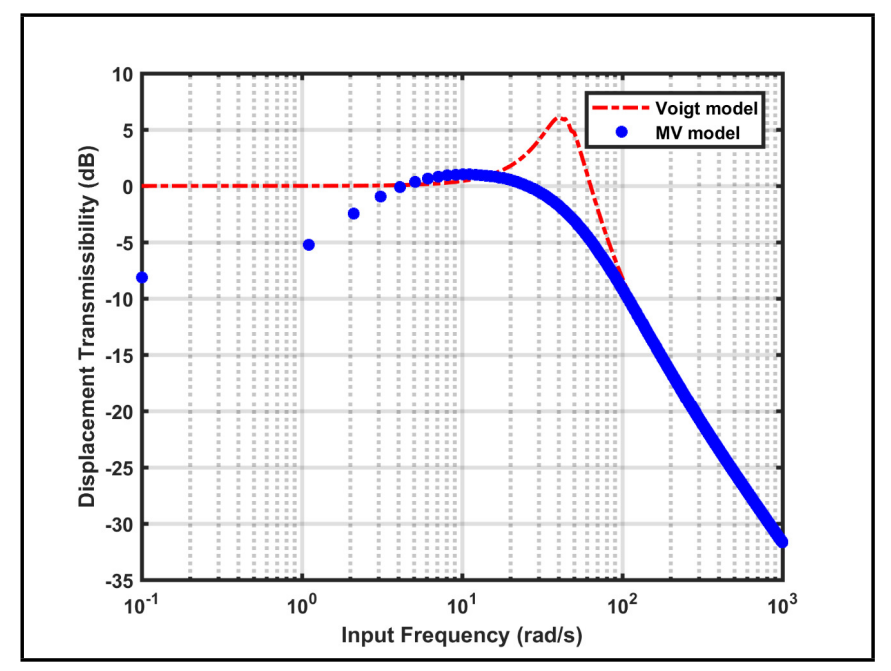

Figure 15. Displacement Transmissibility — Model Comparison.

(QZS) mechanism that has been investigated in the literature. ${ }^{24}$ Direct numerical comparisons with the QZS mechanism have not been performed in this study since the results for both the models depend on a large number of variables. ${ }^{24,25}$ However, the trends exhibited by the models discussed in this section are similar to the QZS models, this includes the ability of the isolation system to mitigate the response over a larger frequency range and the ability of the isolation system to reduce the response at relatively lower frequencies.

As can be seen from the results in Fig. 15, the MV model with horizontal spring elements along $x$ and $z$ axes is able to significantly mitigate the transmissibility at resonance and at lower frequencies, as compared to the Voigt model. However, it is important to note that these results may vary with the choice of variables associated with the MV model. In general, the simulation results have pinpointed the use of precompression and stiffness of the horizontal spring elements as important variables that can be used in the design of the isolation system. This is particularly important for applications that have significantly different stiffness requirements in different planes, as discussed earlier in the paper. The models discussed in this study will be specifically relevant to such applications.

\section{CONCLUSIONS}

In this paper, the effect of stiffness nonlinearity has been investigated by incorporating stiffness elements along nonisolating axes into the MV and MMV models for a vibration isolator. An alternative design of a vibration isolator is investigated in this study with significantly different stiffness properties along multiple axes of the isolator. The main advantage of this design is an ability to control the frequency response over a relatively larger frequency range. Such a design could mitigate some of the trade-offs typically associated with the design of a passive isolator. This design can also be used to accommodate multiple performance constraints posed on an isolation system while requiring the system to effectively mitigate vibration response. These performance constraints are important in applications such as motorcycles where the stiffness requirements of the isolation system are significantly different along the non-isolating axes due to handling and packaging requirements. ${ }^{17}$ A drawback of the proposed design is a significant enhancement of design complexity and related challenges associated with manufacturing the vibration isolator.

Results indicate that the incorporation of stiffness nonlinearity, as investigated in this study, can be useful in enhancing vibration isolation characteristics of a passive isolator while allowing the design to meet other performance criteria that the isolation system may be required to satisfy. Specifically, the stiffness nonlinearity is seen to significantly reduce the response amplitude at lower frequencies with limited trade-offs for relatively higher frequencies. The stiffness of the horizontal spring elements in conjunction with the level of precompression are found to be critical in controlling the frequency response, time response as well as displacement transmissibility. The simulation results do not vary much between the MV and the MMV models, and the MMV model is not seen to exhibit any specific advantages for the models investigated in this study.

As a follow up to this study, the coupled influence of stiffness and damping nonlinearities will be investigated in the future for the models analyzed in this paper. The numerical solution will be investigated further to compute the time response at higher levels of stiffness and to comprehend whether the assumptions associated with the HBM are appropriate for all the configurations investigated in this study. The output frequency response function (OFRF) approach will be used to account for the influence of higher harmonics in future work. ${ }^{2}$ The models discussed in this paper will also be numerically compared to other similar models in the literature such as the QZS model.

\section{REFERENCES}

1 Ibrahim, R. A. Recent Advances in Nonlinear Passive Vibration Isolators, Journal of Sound and Vibration, 314, 371-452, (2008). https://dx.doi.org/10.1016/j.jsv.2008.01.014

2 Ho, C., Lang, Z., and Billings, S. A. Design of vibration isolators by exploiting the beneficial effects of stiffness and damping nonlinearities, Journal of Sound and Vibration, 333, 2489-2504, (2014). https://dx.doi.org/10.1016/j.jsv.2014.02.011 
3 Ravindra, B., and Mallik, A. K. Performance of nonlinear vibration isolators under harmonic excitation, Journal of Sound and Vibration, 170, 325-337, (1994). https://dx.doi.org/10.1006/jsvi.1994.1066

4 Sun, X. and Jing, X. Analysis and design of a nonlinear stiffness and damping system with a scissor-like structure, Mechanical Systems and Signal Processing, 66-67, 723-742, (2016). https://dx.doi.org/10.1016/j.ymssp.2015.05.026

5 Liu, C., Jing, X., and Chen Z. Band stop vibration suppression using a passive $\mathrm{X}$-shape structured lever-type isolation system, Mechanical Systems and Signal Processing, 68-69, 342-353, (2016). https://dx.doi.org/10.1016/j.ymssp.2015.07.018

6 Sun, X., Jing, X., Xu, J., and Cheng, L. Vibration isolation via a scissor-like structured platform, Journal of Sound and Vibration, 333, 2404-2420, (2014). https://dx.doi.org/10.1016/j.jsv.2013.12.025

7 Kaul, S. Maxwell-Voigt and Maxwell Ladder Models for Multi-Degree-of-Freedom Elastomeric Isolation Systems, Journal of Vibration and Acoustics, 137, 021021, (2014). https://dx.doi.org/10.1115/1.4029538

8 Yang, J., Xiong, Y. P., and Xing, J. T. Dynamics and power flow behaviour of a nonlinear vibration isolation system with a negative stiffness mechanism, Journal of Sound and Vibration, 332, 167-183, (2013). https://dx.doi.org/10.1016/j.jsv.2012.08.010

9 Le, T. D. and Ahn, K. K. Experimental investigation of a vibration isolation system using negative stiffness structure, International Journal of Mechanical Sciences, 70, 99-112, (2013). https://dx.doi.org/10.1016/j.ijmecsci.2013.02.009

10 Valeev, A., Zotov, A., and Kharisov, S. Designing of Comapct Low Frequency Vibration Isolator with Quasi-Zero-Stiffness, Journal of Low Frequency Noise, Vibration and Active Control, 34, 459-474, (2015). https://dx.doi.org/10.1260/0263-0923.34.4.459

11 Sun, X. and Jing, X. Multi-direction vibration isolation with quasi-zero stiffness by employing geometrical nonlinearity, Mechanical Systems and Signal Processing, 62-63, 149-163, (2015). https://dx.doi.org/10.1016/j.ymssp.2015.01.026

12 Sun, X. and Jing, X. A nonlinear vibration isolator achieving high-static-low-dynamic stiffness and tunable anti-resonance frequency band, Mechanical Systems and Signal Processing, 80, 166-188, (2016). https://dx.doi.org/10.1016/j.ymssp.2016.04.011

13 Mallik, A. K., Kher, V., Puri, M., and Hatwal, H. On the modelling of Non-linear Elastomeric Vibration Isolators, Journal of Sound and Vibration, 219, 239-253, (1999). https://dx.doi.org/10.1006/jsvi.1998.1883

14 Renaud, F., Dion, J., Chevallier, G., Tawfiq, I., and Lemaire, R. A new identification method of viscoelastic behavior:
Application to the generalized Maxwell model, Mechanical Systems and Signal Processing, 25, 991-1010, (2011). https://dx.doi.org/10.1016/j.ymssp.2010.09.002

15 Hao, D., Li, D., and Liao, Y. A finite viscoelastic constitutive model for filled rubber-like materials, International Journal of Solids and Structures, 64-65, 232-245, (2015). https://dx.doi.org/10.1016/j.ijsolstr.2015.04.002

16 Zhang, W. and Zhao, J. Analysis on nonlinear stiffness and vibration isolation performance of scissor-like structure with full types, Nonlinear Dynamics, 86, 17-36, (2016). https://dx.doi.org/10.1007/s11071-016-2869-z

17 Kaul, S. and Dhingra, A. K. Engine mount optimization for vibration isolation in motorcycles, Vehicle System Dynamics, 47, 419-436, (2009). https://dx.doi.org/10.1080/00423110802167458

18 Lee, J., Ghasemi, A. H., Okwudire, C. E., and Scruggs, J. A Linear Feedback Control Framework for Optimally Locating Passive Vibration Isolators with known Stiffness and Damping Parameters, Journal of Vibration and Acoustics, 139, 011006, (2017). https://dx.doi.org/10.1115/1.4034771

19 Jordan, D. W. and Smith, P. Nonlinear Ordinary Differential Equations, Fourth Edition, Oxford University Press, New York, NY, (2007).

${ }^{20}$ Garcia-Saldana, J. D. and Gasull, A. A finite viscoelastic constitutive model for filled rubber-like materials, Journal of Differential Equations, 254, 67-80, (2013). https://dx.doi.org/10.1016/j.jde.2012.09.011

21 Peng, Z. K., Meng, G., Lang, Z. Q., Zhang, W. M., and Chu, F. L. Study of the effects of cubic nonlinear damping on vibration isolations using Harmonic Balance Method, International Journal of Non-Linear Mechanics, 47, 1073-1080, (2012). https://dx.doi.org/10.1016/j.ijnonlinmec.2011.09.013

22 Tarrago, M. J. G., Gil-Negrete, N., and Vinolas, J. Viscoelastic models for rubber mounts: influence on the dynamic behaviour of an elastomeric isolated system, International Journal of Vehicle Design, 49, 303-317, (2009). https://dx.doi.org/10.1504/ijvd.2009.024960

23 MathWorks, MATLAB User Guide, MathWorks, Natick, MA, (2016).

24 Carrella, A., Brennan, M. J., and Waters, T. P. Static analysis of a passive vibration isolator with quasi-zero-stiffness characteristic, Journal of Sound and Vibration, 301, 678689, (2007). https://dx.doi.org/10.1016/j.jsv.2006.10.011

25 Carrella, A., Brennan, M. J., Kovacic, I., and Waters, T. P. On the force transmissibility of a vibration isolator with quasi-zero-stiffness, Journal of Sound and Vibration, 332, 707-717, (2009). https://dx.doi.org/10.1016/j.jsv.2008.11.034 